\title{
Application of bioinformatics for identification of candidate genes conferring tolerance to drought and salinity in chickpea (Cicer arietinum L.) in the environment of Kazakhstan
}

\author{
G. Khassanova ${ }^{1 *}$, A. Zhubatkanov ${ }^{1}$, S. Jatayev ${ }^{1}$, A. Turbekova ${ }^{1}$, Y. Shavrukov ${ }^{2}$ \\ ${ }^{1}$ S. Seifullin Kazakh AgroTechnical University, Astana, Kazakhstan \\ ${ }^{2}$ Flinders University, Adelaide, Australia \\ *e-mail: khasanova-gulmira@mail.ru
}

Key words: bioinformatics, plant biology, gene expression, chickpea, drought resistance

Introduction and Aim: Chickpea is gaining popularity as a crop in Kazakhstan, with its arid climate and soils with localised, patchy salinity. The two chickpea ecotypes, Desi and Kabuli, differ in various growth and seed production traits and supply diverse markets reflecting differing consumer preferences. Based on the results of field trials, genotypes for both ecotypes were selected for two groups with either high or low seed yield, 1000 seed weight, and lowest pod height. The selected genotypes were used for further bioinformatic and molecular genetic analyses. The aim of this study was to identify and analyse possible candidate genes controlling better tolerance to drought and salinity and test for their association with higher seed yield in selected chickpea genotypes via bioinformatics.

Methods: International and local germplasm collections comprising 250 chickpea samples were tested over two years in field trials in Northern and Central Kazakhstan. Additional experiments with drought and salinity treatments were carried out in greenhouses. Bioinformatics and systems biology methods were applied in this study to identify important candidate genes and their possible involvement in tolerance to abiotic stresses and seed yield in the selected chickpea genotypes.

Results: Eight possible candidate genes encoding various structural and regulatory polypeptides were identified in chickpea using bioinformatic analyses with comparisons to other plant species. Expression analyses revealed the maximum level of expression in $C a R A B C 1$, which controls cell membrane trafficking and is associated with better adaptation of plants to abiotic stress conditions. Different levels of expression were found between the two groups of chickpea genotypes with high and low seed yield, the two ecotypes and following exposure to drought or salinity stress. CaRABCl expression seems to be regulated by Transcription factor $C a D R E B 2$, but this hypothesis will need to be verified by further molecular experiments.

Conclusion: The candidate gene CaRABC1, possibly regulated by CaDREB2, was identified with the use of bioinformatic and molecular genetic analyses. The genetic polymorphism of these candidate genes can be used for molecular breeding of chickpea genotypes with high seed yield and better adaptation to the environment of Kazakhstan. Acknowledgements: This study was supported by the Ministry of Education and Science. Kazakhstan, Research program BR05236500. 